\title{
Infinite Series Representations of the Trivariate and Quadrivariate Rayleigh Distribution and Their Applications
}

\author{
Yunxia Chen, Student Member, IEEE, and Chinthananda Tellambura, Senior Member, IEEE
}

\begin{abstract}
Few theoretical results are known about the joint distribution of three or more arbitrarily correlated Rayleigh random variables (RVs). Consequently, theoretical performance results are unknown for three- and four-branch equal gain combining (EGC), selection combining (SC), and generalized SC (GSC) in correlated Rayleigh fading. This paper redresses this gap by deriving new infinite series representations for the joint probability density function (pdf) and the joint cumulative distribution function (cdf) of three and four correlated Rayleigh RVs. Bounds on the error resulting from truncating the infinite series are derived. A classical approach, due to Miller, is used to derive our results. Unfortunately, Miller's approach cannot be extended to more than four variates and, in fact, the quadrivariate case considered in this paper appears to be the most general result possible. For brevity, we treat only a limited number of applications in this paper. The new pdf and cdf expressions are used to derive the outage probability of three-branch SC, the moments of the EGC output signal-to-noise ratio (SNR), and the moment generating function of the GSC $(2,3)$ output SNR in arbitrarily correlated Rayleigh fading. A novel application of Bonferroni's inequalities allows new outage bounds for multibranch $\mathrm{SC}$ in arbitrarily correlated Rayleigh channels.
\end{abstract}

Index Terms-Characteristic function (chf), diversity, moment generating function (mgf), quadrivariate Rayleigh distribution, trivariate Rayleigh distribution.

\section{INTRODUCTION}

A S RAYLEIGH fading is frequently used to model the received signal amplitudes in urban and suburban areas [1], the joint probability density function (pdf) of a set of $L$ correlated Rayleigh signals is required for several performance-analysis problems. It is well known that this is an exceedingly difficult problem and, indeed, some authors claim that the joint pdf for more than two correlated Rayleigh envelopes cannot be found [2]. However, since the envelopes of multiple correlated complex Gaussian random variables (RVs) are Rayleigh

Paper approved by V. A. Aalo, the Editor for Diversity and Fading Channel Theory of the IEEE Communications Society. Manuscript received October 24, 2003; revised November 12, 2004 and April 4, 2005. This work was supported in part by the National Sciences and Engineering Research Council (NSERC) and in part by the Alberta Informatics Circle of Research Excellence (iCORE), Canada.

Y. Chen was with the Department of Electrical and Computer Engineering, University of Alberta, Edmonton, AB T6G 2V4, Canada. She is now with the Department of Electrical and Computer Engineering, University of California, Davis, CA 95616 USA (e-mail: yxchen@ece.ucdavis.edu).

C. Tellambura is with the Department of Electrical and Computer Engineering, University of Alberta, Edmonton, AB T6G 2V4, Canada (e-mail: chintha@ece.ualberta.ca).

Digital Object Identifier 10.1109/TCOMM.2005.860059 distributed, the underlying complex Gaussian joint pdf can be converted to polar form to give the joint pdf of amplitudes and phases and, in principle, the phase terms can be integrated out to give the joint amplitude pdf. Following this approach, Miller [3] derives an infinite series of products of modified Bessel functions for the joint pdf of three correlated Rayleigh RVs. While this holds for arbitrary correlation models, it is intractable to derive the joint pdf for $L>3$ using this approach. All the available results therefore treat restricted correlation models. For example, Blumenson and Miller [4] derive the joint pdf of $L$ correlated Rayleigh RVs, provided the inverse covariance matrix $\boldsymbol{\Phi}$ of the underlying Gaussian RVs is tridiagonal (i.e., $\phi_{i j}=0$ if $|i-j|>1$ ). The exponential correlation model generates this particular pattern.

The joint pdf of Rayleigh RVs has many applications, which include determining the impact of correlation on diversity systems and modeling fading processes [2], [5]-[8]. The pdf of bivariate correlated Rayleigh distribution has been derived by Rice [9]. Tan and Beaulieu [10] derive an infinite series representation for this bivariate joint cumulative distribution function (cdf). Consequently, many published papers treating selection combining (SC) and equal gain combining (EGC) in correlated Rayleigh fading are limited to the dual-branch case [2], [11]-[13]. Using Blumenson and Miller's result [4], Karagiannidis et al. derive a joint distribution that holds only for exponentially correlated Nakagami fading channels [14], [15], and apply their results with Green's matrix to approximate arbitrarily correlated fading [16], [17]. However, their approach may not work for the constant correlation case.

In this paper, we use Miller's result [3] to derive new infinite series representations for the joint pdf, cdf, moments, and characteristic function (chf) of three arbitrarily correlated Rayleigh RVs. For four correlated Rayleigh RVs, we generalize Blumenson and Miller's result [4] (which is limited to tridiagonal inverse covariance matrices) to the case where the inverse covariance has five nonzero diagonals (i.e., only $\phi_{14}$ needs to be zero). Our trivariate and quadrivariate cdf series generalize Tan and Beaulieu's series for the bivariate Rayleigh cdf [10]. Although there are many applications of the new pdf and cdf expressions, three- and four-branch diversity systems are particularly of interest. For diversity systems in arbitrarily correlated Rayleigh channels, we derive the outage probability of three-branch SC, Bonferroni upper and lower bounds for the output complementary cdf (ccdf) of $L$-branch $(L>3) \mathrm{SC}$, moments of the three-branch EGC output signal-to-noise ratio (SNR), and the output moment generating function (mgf) of 
the generalized SC (GSC) $(2,3)$ receiver. For brevity, additional results are omitted here, but a detailed application of our results for three-branch SC performance can be found in [18].

This paper is organized as follows. Section II derives new infinite series representations for the joint pdf, cdf, moments, and chf of the trivariate Rayleigh distribution. Bounds on the truncation error are also derived. Section III derives infinite series representations for the joint pdf and cdf of a certain class of the quadrivariate Rayleigh distribution. Section IV presents some applications of the new results. Section V provides several numerical and simulation results. Section VI concludes this paper.

\section{TRIVARIATE DISTRIBUTION}

Let $G=\left\{G_{1}, G_{2}, G_{3}\right\}$ be joint complex Gaussian RVs with zero means and positive definite covariance matrix $\boldsymbol{\Psi}$, whose element is defined as $\psi_{j k}=E\left(G_{j} G_{k}^{*}\right)$, where $E(X)$ is the mean value of $X$. We may write $G_{k}$ in terms of polar coordinates as

$$
G_{k}=R_{k} \exp \left(i \Theta_{k}\right), \quad k \in\{1,2,3\}
$$

where $R_{k}=\left|G_{k}\right|$ is the envelope of $G_{k}$. Thus, $R=$ $\left\{R_{1}, R_{2}, R_{3}\right\}$ is a set of Rayleigh RVs, and $\boldsymbol{\Theta}=\left\{\Theta_{1}, \Theta_{2}, \Theta_{3}\right\}$ is a set of jointly distributed phases. The joint pdf $p_{\boldsymbol{R}, \boldsymbol{\Theta}}(\boldsymbol{r}, \boldsymbol{\theta})$ of $\boldsymbol{R}$ and $\boldsymbol{\Theta}$ can be related to the density $p_{\boldsymbol{G}}(\boldsymbol{g})$ of $\boldsymbol{G}$. Hence, the marginal density $p_{\boldsymbol{R}}(\boldsymbol{r})$ can be obtained by integrating out $p_{\boldsymbol{R}, \boldsymbol{\Theta}}(\boldsymbol{r}, \boldsymbol{\theta})$ over $\boldsymbol{\theta}$. This approach yields [3], [19]

$$
\begin{aligned}
p_{\boldsymbol{R}}\left(r_{1}, r_{2}, r_{3}\right)= & 8 \operatorname{det}(\boldsymbol{\Phi}) r_{1} r_{2} r_{3} e^{-\left(r_{1}^{2} \phi_{11}+r_{2}^{2} \phi_{22}+r_{3}^{2} \phi_{33}\right)} \\
& \times \sum_{k=0}^{\infty} \varepsilon_{k}(-1)^{k} \cos (k \chi) I_{k}\left(2 r_{1} r_{2}\left|\phi_{12}\right|\right) \\
& \times I_{k}\left(2 r_{2} r_{3}\left|\phi_{23}\right|\right) I_{k}\left(2 r_{3} r_{1}\left|\phi_{31}\right|\right)
\end{aligned}
$$

where $\varepsilon_{k}$ is the Neumann factor $\left(\varepsilon_{0}=1, \varepsilon_{k}=2\right.$ for $k=$ $1,2, \ldots)$ and

$$
\chi=\chi_{12}+\chi_{23}+\chi_{31}
$$

and $\boldsymbol{\Phi}$ is the inverse covariance matrix

$$
\boldsymbol{\Phi}=\boldsymbol{\Psi}^{-1}=\left[\begin{array}{lll}
\phi_{11} & \phi_{12} & \phi_{13} \\
\phi_{12}^{*} & \phi_{22} & \phi_{23} \\
\phi_{13}^{*} & \phi_{23}^{*} & \phi_{33}
\end{array}\right], \quad \phi_{j k}=\left|\phi_{j k}\right| e^{i \chi_{j k}}
$$

where $i=\sqrt{-1}$. Based on (2), we next derive the infinite series representations for the joint pdf, cdf, moments, and chf of the trivariate Rayleigh distribution. New bounds on the truncation error of the infinite series are also developed.

\section{A. Joint $p d f$ and Joint $c d f$}

The expression (2) is not very useful, as it is not in a product form of the variables $r_{1}, r_{2}$, and $r_{3}$. Representing the modified Bessel function by an infinite series [20, eq. (9.6.10)], we readily obtain an infinite series representation for the joint pdf as

$$
\begin{aligned}
& p_{\boldsymbol{R}}\left(r_{1}, r_{2}, r_{3}\right) \\
& \quad=8 \operatorname{det}(\boldsymbol{\Phi}) e^{-\left(r_{1}^{2} \phi_{11}+r_{2}^{2} \phi_{22}+r_{3}^{2} \phi_{33}\right)}
\end{aligned}
$$

$$
\begin{aligned}
& \times \sum_{k=0}^{\infty} \varepsilon_{k}(-1)^{k} \cos (k \chi) \\
& \times \sum_{\substack{l, m, n=0 \\
\times}}^{\infty} \frac{\left|\phi_{12}\right|^{2 l+k}}{l !(l+k) !} \frac{\left|\phi_{23}\right|^{2 m+k}}{m !(m+k) !} \frac{\left|\phi_{31}\right|^{2 n+k}}{n !(n+k) !} \\
& \times r_{1}^{2(l+n+k)+1} r_{2}^{2(l+m+k)+1} r_{3}^{2(m+n+k)+1} .
\end{aligned}
$$

Note that (5) is in a product form of $r_{1}, r_{2}, r_{3}$, and is therefore suitable for our purposes. Using the definition of the incomplete gamma function [20], we obtain the infinite series representation for the joint trivariate cdf as

$$
\begin{aligned}
F_{\boldsymbol{R}}\left(\lambda_{1}, \lambda_{2}, \lambda_{3}\right)= & \frac{\operatorname{det}(\boldsymbol{\Phi})}{\phi_{11} \phi_{22} \phi_{33}} \sum_{k=0}^{\infty} \varepsilon_{k}(-1)^{k} \cos (k \chi) \\
& \times \sum_{l, m, n=0}^{\infty} C \nu_{12}^{l+\frac{k}{2}} \nu_{23}^{m+\frac{k}{2}} \nu_{31}^{n+\frac{k}{2}} \gamma\left(\delta_{1}, \lambda_{1}^{2} \phi_{11}\right) \\
& \times \gamma\left(\delta_{2}, \lambda_{2}^{2} \phi_{22}\right) \gamma\left(\delta_{3}, \lambda_{3}^{2} \phi_{33}\right)
\end{aligned}
$$

where $\chi$ is defined as (3) and

$$
\begin{aligned}
C & =\frac{1}{l !(l+k) ! m !(m+k) ! n !(n+k) !} \\
\nu_{j k} & =\frac{\left|\phi_{j k}\right|^{2}}{\phi_{j j} \phi_{k k}} \\
\delta_{1} & =l+n+k+1 \\
\delta_{2} & =m+l+k+1 \\
\delta_{3} & =n+m+k+1 .
\end{aligned}
$$

The above notations (3) and (7) will be used throughout this paper for brevity. The cdf (6) holds for any arbitrary $3 \times 3$ correlation matrix. Let us consider two commonly used spatial correlation models.

1) Constant Correlation Model: The constant correlation model is valid for a set of closely placed antennas [21]. The normalized correlation matrix of this model is given by $\psi_{j k}=\rho(j \neq k)$ and $\psi_{j j}=1$, where $-(1 / 2) \leq \rho<1$. It can be shown that $\chi=\chi_{12}+\chi_{23}+\chi_{31}=3 \pi$. Thus, the joint cdf (6) reduces to

$$
\begin{aligned}
F_{\boldsymbol{R}}\left(\lambda_{1}, \lambda_{2}, \lambda_{3}\right) & \\
= & \frac{(1-\rho)(1+2 \rho)^{2}}{(1+\rho)^{3}} \\
& \times \sum_{k=0}^{\infty} \varepsilon_{k} \sum_{l, m, n=0}^{\infty} C\left(\frac{\rho}{1+\rho}\right)^{\delta_{1}+\delta_{2}+\delta_{3}-3} \\
& \times \gamma\left(\delta_{1}, \frac{(1+\rho) \lambda_{1}^{2}}{1+\rho-2 \rho^{2}}\right) \gamma\left(\delta_{2}, \frac{(1+\rho) \lambda_{2}^{2}}{1+\rho-2 \rho^{2}}\right) \\
& \times \gamma\left(\delta_{3}, \frac{(1+\rho) \lambda_{3}^{2}}{1+\rho-2 \rho^{2}}\right) .
\end{aligned}
$$

2) Exponential Correlation Model: The exponential correlation model can be used to describe the correlation among equally spaced linear antenna arrays [14]. The normalized correlation matrix of this model is described as $\psi_{j k}=\rho^{|j-k|}$, 
TABLE I

Number of Terms NeEded In (8) To AChiEve Five-SignificAnT-Figure ACCuracy. $\lambda_{1}=\lambda_{2}=\lambda_{3}=\lambda$

\begin{tabular}{|c|c|c|c|}
\hline & $\lambda=1$ & $\lambda=3$ & $\lambda=5$ \\
\hline$\rho=0.1$ & $K=L=M=N=2$ & $K=2, L=M=N=3$ & $K=L=M=N=3$ \\
\hline$\rho=0.3$ & $K=2, L=M=N=3$ & $K=5, L=M=N=6$ & $K=L=M=N=6$ \\
\hline$\rho=0.5$ & $K=3, L=M=N=4$ & $K=7, L=M=N=8$ & $K=L=M=N=8$ \\
\hline
\end{tabular}

where $0 \leq \rho<1$. It can then be shown that $\phi_{31}=\phi_{13}=0$. Thus, the joint cdf can be simplified considerably to

$$
\begin{aligned}
& F_{\boldsymbol{R}}\left(\lambda_{1}, \lambda_{2}, \lambda_{3}\right) \\
& =\frac{1-\rho^{2}}{1+\rho^{2}} \sum_{l, m=0}^{\infty} \frac{1}{(l !)^{2}(m !)^{2}}\left(\frac{\rho^{2}}{1+\rho^{2}}\right)^{l+m} \\
& \quad \times \gamma\left(l+1, \frac{\lambda_{1}^{2}}{1-\rho^{2}}\right) \gamma\left(l+m+1, \frac{\left(1+\rho^{2}\right) \lambda_{2}^{2}}{1-\rho^{2}}\right) \\
& \quad \times \gamma\left(m+1, \frac{\lambda_{3}^{2}}{1-\rho^{2}}\right)
\end{aligned}
$$

which is equivalent to [14, eq. (6)].

\section{B. Truncation Error}

Assume that the cdf series (6) is limited to $K, L, M$, and $N$ terms in the variables $k, l, m$, and $n$, respectively. The remaining terms constitute the truncation error. It is desirable to obtain a simple bound for the truncation error, because such a bound is useful for studying the impact of correlation $\Psi$ on the truncation error. Unfortunately, this is not always possible. For example, Tan and Beaulieu [10] derive a truncation error bound in terms of the confluent hypergeometric functions, where the relationship between the correlation $\rho$ and the truncation error is not immediately evident. Here, we provide an alternative method to bound the truncation error.

We notice that the incomplete gamma function $\gamma(a, x) \leq$ $\Gamma(a)$ and $|\cos (\chi)| \leq 1$. The truncation error of (6) can therefore be upper bounded by

$$
\begin{aligned}
\left|E_{T}\right| \leq & \sum_{k=K}^{\infty} \sum_{l=0}^{\infty} \sum_{m=0}^{\infty} \sum_{n=0}^{\infty} G(k, l, m, n) \\
& +\sum_{k=0}^{K-1} \sum_{l=L}^{\infty} \sum_{m=0}^{\infty} \sum_{n=0}^{\infty} G(k, l, m, n) \\
& +\sum_{k=0}^{K-1} \sum_{l=0}^{L-1} \sum_{m=M}^{\infty} \sum_{n=0}^{\infty} G(k, l, m, n) \\
& +\sum_{k=0}^{K-1} \sum_{l=0}^{L-1} \sum_{m=0}^{M-1} \sum_{n=N}^{\infty} G(k, l, m, n)
\end{aligned}
$$

where

$$
\begin{array}{r}
G(k, l, m, n)=\frac{\operatorname{det}(\boldsymbol{\Phi})}{\phi_{11} \phi_{22} \phi_{33}} \varepsilon_{k}\left(\begin{array}{c}
l+n+k \\
l
\end{array}\right)\left(\begin{array}{c}
l+m+k \\
m
\end{array}\right) \\
\times\left(\begin{array}{c}
m+n+k \\
n
\end{array}\right) \nu_{12}^{l+\frac{k}{2}} \nu_{23}^{m+\frac{k}{2}} \nu_{31}^{n+\frac{k}{2}}
\end{array}
$$

Further simplification of (10) is complicated. Due to the space limitation, we only provide an upper bound for the truncation error in the special case of exponentially correlated models where $\phi_{13}=0$. This leads to

$$
\begin{aligned}
&\left|E_{T}\right| \leq \frac{1-\rho^{2}}{1+\rho^{2}} {\left[\sum_{l=L}^{\infty} \sum_{m=0}^{\infty}\left(\begin{array}{c}
l+m \\
m
\end{array}\right)\left(\frac{\rho^{2}}{1+\rho^{2}}\right)^{l+m}\right.} \\
&\left.+\sum_{l=0}^{L-1} \sum_{m=M}^{\infty}\left(\begin{array}{c}
l+m \\
m
\end{array}\right)\left(\frac{\rho^{2}}{1+\rho^{2}}\right)^{l+m}\right] \\
& \leq 1-\left(1-\rho^{4}\right)\left[1-\left(\frac{\rho^{2}}{1+\rho^{2}}\right)^{L}\right]\left[1-\left(\frac{\rho^{2}}{1+\rho^{2}}\right)^{M}\right] .
\end{aligned}
$$

As expected, the upper bound for the truncation error increases as $\rho$ increases, and decreases as the number of terms increases. However, compared with the bounds developed in [14], our bounds (10) may be loose for small values of $\lambda_{k}$ 's in (6).

The converge rate of (6) depends not only on the correlation $\Psi$ but also on the values of $\lambda_{k}$ 's. For brevity, we consider the constant correlation model here. Table I lists the number of the terms required in (8) to achieve five-significant-figure accuracy. As $\lambda_{k}$ 's increase, more terms are needed in the cdf series, i.e., the truncation error increases for the same number of terms.

\section{Joint Moments and Joint chf}

Moments and chf are often used to characterize the RVs. Using the infinite series representation for the joint trivariate pdf (5), we derive the joint moments and the joint chf of three Rayleigh RVs.

If $\alpha, \beta, \theta>-2$, the joint moments are given by

$$
\begin{aligned}
E\left(r_{1}^{\alpha} r_{2}^{\beta} r_{3}^{\theta}\right)= & \frac{\operatorname{det}(\boldsymbol{\Phi})}{\phi_{11}^{1+\alpha / 2} \phi_{22}^{1+\beta / 2} \phi_{33}^{1+\theta / 2}} \sum_{k=0}^{\infty} \varepsilon_{k}(-1)^{k} \cos (k \chi) \\
& \times \sum_{l, m, n=0}^{\infty} C \nu_{12}^{l+k / 2} \nu_{23}^{m+k / 2} \nu_{31}^{n+k / 2} \Gamma\left(\delta_{1}+\frac{\alpha}{2}\right) \\
& \times \Gamma\left(\delta_{2}+\frac{\beta}{2}\right) \Gamma\left(\delta_{3}+\frac{\theta}{2}\right)
\end{aligned}
$$

where $\Gamma(a)$ is the gamma function [20]. The joint moments have many applications. We will show that statistical moments of the output SNR of certain diversity systems can be evaluated using (13).

The joint chf of the trivariate Rayleigh distribution is defined as the statistical average

$$
\begin{aligned}
\phi\left(v_{1}, v_{2}, v_{3}\right)= & E\left[e^{i\left(v_{1} r_{1}+v_{2} r_{2}+v_{3} r_{3}\right)}\right] \\
= & \frac{\operatorname{det} \boldsymbol{\Phi}}{\phi_{11} \phi_{22} \phi_{33}} \sum_{k=0}^{\infty} \varepsilon_{k}(-1)^{k} \cos (k \chi) \\
& \times \sum_{l, m, n=0}^{\infty} C \nu_{12}^{l+k / 2} \nu_{23}^{m+k / 2} \nu_{31}^{n+k / 2}
\end{aligned}
$$




$$
\begin{aligned}
& \times \exp \left[-\left(\frac{v_{1}^{2}}{8 \phi_{11}}+\frac{v_{2}^{2}}{8 \phi_{22}}+\frac{v_{3}^{2}}{8 \phi_{33}}\right)\right] \\
& \times \frac{\left(2 \delta_{1}-1\right) !\left(2 \delta_{2}-1\right) !\left(2 \delta_{3}-1\right) !}{2^{\delta_{1}+\delta_{2}+\delta_{3}-3}} \\
& \times D_{-2 \delta_{1}}\left(\frac{-i v_{1}}{\sqrt{2 \phi_{11}}}\right) D_{-2 \delta_{2}}\left(\frac{-i v_{2}}{\sqrt{2 \phi_{22}}}\right) \\
& \times D_{-2 \delta_{3}}\left(\frac{-i v_{3}}{\sqrt{2 \phi_{33}}}\right)
\end{aligned}
$$

where $D_{-n}(z)$ is the parabolic cylinder function [30].

\section{QUADRIVARIATE RAYLEIGH DISTRIBUTION}

Blumenson and Miller [4] derive the joint pdf and the joint cdf of the multivariate Rayleigh distribution. However, their expression is only valid when the inverse covariance matrix satisfies $\phi_{j k}=0$ for $|j-k|>1$ (i.e., for the matrix in (15), both $\phi_{13}$ and $\phi_{24}$ would be zero). However, we can derive more general results than [4] for the quadrivariate case. We consider the inverse covariance matrix given by

$$
\boldsymbol{\Phi}=\Psi^{-1}=\left[\begin{array}{cccc}
\phi_{11} & \phi_{12} & \phi_{13} & 0 \\
\phi_{12}^{*} & \phi_{22} & \phi_{23} & \phi_{24} \\
\phi_{13}^{*} & \phi_{23}^{*} & \phi_{33} & \phi_{34} \\
0 & \phi_{24}^{*} & \phi_{34}^{*} & \phi_{44}
\end{array}\right], \quad \phi_{j k}=\left|\phi_{j k}\right| e^{i \chi_{j k}}
$$

Using the relation between complex Gaussian RVs and Rayleigh RVs, we show that the joint pdf of four Rayleigh RVs, $\boldsymbol{R}=$ $\left\{R_{1}, R_{2}, R_{3}, R_{4}\right\}$, with positive definite $\boldsymbol{\Psi}$ and its $\boldsymbol{\Phi}$ satisfying (15) can be obtained as a product of the modified Bessel functions of the first kind (see Appendix A)

$$
\begin{aligned}
p_{\boldsymbol{R}}\left(r_{1}, r_{2}, r_{3}, r_{4}\right) \\
=16 \operatorname{det}(\boldsymbol{\Phi}) r_{1} r_{2} r_{3} r_{4} e^{-\left(r_{1}^{2} \phi_{11}+r_{2}^{2} \phi_{22}+r_{3}^{2} \phi_{33}+r_{4}^{2} \phi_{44}\right)} \\
\quad \times \sum_{j=0}^{\infty} \sum_{k=-\infty}^{\infty} \varepsilon_{j}(-1)^{j+k} \\
\quad \times \cos \left[j\left(\chi_{12}+\chi_{23}+\chi_{31}\right)+k\left(\chi_{23} \chi_{34}+\chi_{42}\right)\right] \\
\quad \times I_{j}\left(2 r_{1} r_{2}\left|\phi_{12}\right|\right) I_{j}\left(2 r_{1} r_{3}\left|\phi_{13}\right|\right) \\
\quad \times I_{k}\left(2 r_{2} r_{4}\left|\phi_{24}\right|\right) I_{k}\left(2 r_{3} r_{4}\left|\phi_{34}\right|\right) \\
\quad \times I_{j+k}\left(2 r_{2} r_{3}\left|\phi_{23}\right|\right) .
\end{aligned}
$$

To the best of our knowledge, (16) is a novel result which allows evaluation of four-branch diversity combiners in several kinds of correlated fading channels. Equation (16) reduces to previous results for two special cases. We believe that an expression akin to (16) cannot be derived unless $\phi_{14}=0$. Thus, this appears to be the most general quadrivariate case that yields an infinite series solution. Moreover, if $\phi_{14}$ is not zero in a given application, we may choose a "best" approximation $\widehat{\Psi}$ of $\boldsymbol{\Psi}$ satisfying (15), as follows. We can use a constrained least-squares approach by minimizing $\|\Psi-\widehat{\Psi}\|^{2}$, subject to the constraint that $\left[\widehat{\Psi}^{-1}\right]_{3,4}=0$. A similar problem is discussed in [16].

1) Independent Rayleigh Envelopes: Since the covariance matrix $\Psi$ for independent Rayleigh RVs is diagonal, the inverse covariance matrix $\boldsymbol{\Phi}$ is also diagonal, which is given by $\phi_{j k}=0(j \neq k)$ and $\phi_{j j}=1$ for $j, k \in\{1, \ldots, 4\}$. Therefore, our new expression (16) can be simplified to

$p_{\boldsymbol{R}}\left(\lambda_{1}, \lambda_{2}, \lambda_{3}, \lambda_{4}\right)=\frac{16 r_{1} r_{2} r_{3} r_{4}}{\psi_{11} \psi_{22} \psi_{33} \psi_{44}} e^{-\left(\frac{r_{1}^{2}}{\psi_{11}}+\frac{r_{2}^{2}}{\psi_{22}}+\frac{r_{3}^{2}}{\psi_{33}}+\frac{r_{4}^{2}}{\psi_{44}}\right)}$

which is the product of four independent Rayleigh pdfs [22, eq. (2-1-128)].

2) Exponentially Correlated Rayleigh Envelopes: Substituting $\phi_{24}=\phi_{13}=0$ into (16), we obtain the joint pdf of the exponentially correlated quadrivariate Rayleigh distribution

$$
\begin{aligned}
& p_{\boldsymbol{R}}\left(r_{1}, r_{2},\right.\left.r_{3}, r_{4}\right)=\frac{16 r_{1} r_{2} r_{3} r_{4}}{\left(1-\rho^{2}\right)^{3}} e^{-\left(\frac{r_{1}^{2}+r_{4}^{2}}{1-\rho^{2}}+\frac{\left(1+\rho^{2}\right)\left(r_{2}^{2}+r_{3}^{2}\right)}{1-\rho^{2}}\right)} \\
& \times I_{0}\left(\frac{2 r_{1} r_{2} \rho}{1-\rho^{2}}\right) I_{0}\left(\frac{2 r_{3} r_{4} \rho}{1-\rho^{2}}\right) I_{0}\left(\frac{2 r_{2} r_{3} \rho}{1-\rho^{2}}\right)
\end{aligned}
$$

where $\rho=\psi_{12}$. This expression is equivalent to the previous result [14, eq. (3)].

Expanding $I_{m}(x)$ in infinite series and integrating (16) yield an infinite series representation for the corresponding joint cdf

$$
\begin{aligned}
F_{\boldsymbol{R}}( & \left.\lambda_{1}, \lambda_{2}, \lambda_{3}, \lambda_{4}\right) \\
= & \frac{\operatorname{det}(\boldsymbol{\Phi})}{\phi_{11} \phi_{22} \phi_{33} \phi_{44}} \sum_{j=0}^{\infty} \sum_{k=-\infty}^{\infty} \varepsilon_{j}(-1)^{j+k} \cos \omega \\
& \times \sum_{l, m, n, u=0}^{\infty} \nu_{12}^{l+\frac{j}{2}} \nu_{13}^{m+\frac{j}{2}} \nu_{24}^{n+\frac{|k|}{2}} \nu_{34}^{u+\frac{|k|}{2}} \\
& \times \frac{\gamma\left(u+|k|+n+1, \lambda_{4}^{2} \phi_{44}\right)}{n ! u !(u+|k|) !(n+|k|) !} \frac{\gamma\left(l+j+m+1, \lambda_{1}^{2} \phi_{11}\right)}{l ! m !(l+j) !(m+j) !} \\
& \times \sum_{v=0}^{\infty} \nu_{23}^{v+\frac{|j+k|}{2}} \frac{1}{v !\left(v+\frac{|j+k|}{2}\right) !} \\
& \times \gamma\left(m+n+v+\tau+1, \lambda_{2}^{2} \phi_{22}\right) \\
& \times \gamma\left(m+u+v+\tau+1, \lambda_{3}^{2} \phi_{33}\right)
\end{aligned}
$$

where $\omega=j\left(\chi_{12}+\chi_{23}+\chi_{31}\right)+k\left(\chi_{23}+\chi_{34}+\chi_{42}\right)$ and $\tau=(|j+k|+|k|+j) / 2$. We observe that both (5) and (16) are series of the product of the modified Bessel functions. For brevity, we only discuss the trivariate Rayleigh distribution and its applications in the rest of this paper. Similar results can be obtained for the special class of quadrivariate Rayleigh distribution.

\section{APPLICATIONS}

The new results developed in Section II enable the performance analysis of three-branch diversity systems in arbitrarily correlated Rayleigh fading channels. This section presents four possible applications.

\section{A. Outage Probability of Three-Branch SC}

Outage probability is a standard and widely used performance measure of diversity systems. It is defined as the probability that the output instantaneous SNR $\gamma$ falls below a certain given threshold $\gamma_{\text {th }}$. For independent fading, outage expressions have 
been fully developed [23]. This is not, however, true for correlated fading. Here, we use the joint trivariate Rayleigh cdf (6) to evaluate the outage probability of three-branch SC in correlated fading channels.

We assume that the noise components at different diversity branches are additive white Gaussian noise (AWGN) with identical power spectral density. Let $\gamma_{k}$ and $\bar{\gamma}_{k}$ denote the instantaneous and the average SNR at the $k$ th branch $(k=1,2,3)$. In SC, the branch with the largest instantaneous SNR is selected as the output, $\gamma_{\mathrm{sc}}=\max \left(\gamma_{1}, \gamma_{2}, \gamma_{3}\right)$. Using the relation $\gamma_{k}=\left(\bar{\gamma}_{k} / E\left(r_{k}^{2}\right)\right) r_{k}^{2}=\left(\bar{\gamma}_{k} / \psi_{k k}\right) r_{k}^{2}$, where $r_{k}$ is the amplitude of the received signal at the $k$ th branch, we may obtain the outage probability as

$$
\begin{aligned}
P_{\mathrm{out}} & =\operatorname{Pr}\left(0 \leq \gamma_{\mathrm{sc}} \leq \gamma_{\mathrm{th}}\right) \\
& =F\left(\sqrt{\frac{\gamma_{\mathrm{th}} \psi_{11}}{\overline{\gamma_{1}}}}, \sqrt{\frac{\gamma_{\mathrm{th}} \psi_{22}}{\overline{\gamma_{2}}}}, \sqrt{\frac{\gamma_{\mathrm{th}} \psi_{33}}{\overline{\gamma_{3}}}}\right)
\end{aligned}
$$

where $F_{\boldsymbol{R}}\left(\lambda_{1}, \lambda_{2}, \lambda_{3}\right)$ is the joint cdf of the branch amplitudes (6). Note that the covariance matrix $\Psi$ specifies the correlation (fading correlation) between two complex Gaussian samples. The relationship between the envelope correlation (i.e., the correlation between the two Rayleigh samples) and the fading correlation can be found in [6, eq. (1.5-26)]. Thus, the outage can be evaluated in terms of envelope correlation and the average branch SNRs.

\section{B. Bounds for the Output ccdf of Multibranch SC}

Performance of multibranch SC $(L>3)$ is completely known for independent fading branches. If, however, branch signals are allowed to be correlated (which is a very realistic assumption), known theoretical results are few and far between. In [24] and [25], the performance of multibranch SC in a correlated Rayleigh fading channel is analyzed. However, their results are fairly complicated for large $L(>3)$. From both practical and theoretical standpoints, performance bounds for multibranch SC are therefore desirable. For this problem in its most general setting, we need to know the $L$ th-order joint distribution of the instantaneous branch SNRs for any correlation structure. As mentioned before, unfortunately, it is extremely difficult to derive this joint pdf for $L>3$. Using (5) and (6), we can handle any arbitrary correlation pattern for $L=3$. Can we use our new results to obtain the performance bounds of multibranch SC for $L>3$ ? Strangely enough, the answer is yes. For this purpose, we need to use the Boole formula, which shows that the probability that at least one of the $L$ events $\left\{A_{1}, A_{2}, \ldots, A_{L}\right\}$ occurs is given by [26]

$$
\begin{aligned}
\operatorname{Pr}\left(\bigcup_{u=1}^{L} A_{u}\right)= & \underbrace{\sum_{u}^{L} \operatorname{Pr}\left(A_{u}\right)}_{S_{1}}-\underbrace{\sum_{\substack{u, v=1 \\
u<v}}^{L} \operatorname{Pr}\left(A_{u} \cap A_{v}\right)}_{S_{2}} \\
& +\underbrace{\sum_{\substack{u, v, w=1 \\
u<v<w}}^{L} \operatorname{Pr}\left(A_{u} \cap A_{v} \cap A_{w}\right)-\cdots}_{S_{3}} \\
& +\underbrace{}_{(-1)^{L-1} \operatorname{Pr}\left(A_{1} \cap A_{2} \cap \cdots \cap A_{L}\right) .}
\end{aligned}
$$

Note that $S_{1}$ is the sum of the first-order event probabilities, ignoring any dependency among the events. However, $S_{2}$ is the sum of the pairwise event probabilities, which takes into consideration pairwise dependencies among the events. Using the fact that the sum of an even (or odd) number of terms on the right-hand side of (21) provides a lower (or upper) bound of $\operatorname{Pr}\left(\bigcup_{u=1}^{L} A_{u}\right)$, we obtain the Bonferroni inequalities, of which the second is

$$
S_{1}-S_{2} \leq \operatorname{Pr}\left(\bigcup_{u=1}^{L} A_{u}\right) \leq S_{1}-S_{2}+S_{3} .
$$

We are now in a position to apply this to evaluate the performance of multibranch SC in correlated Rayleigh fading channels. Let $A_{u}$ denote the event that the instantaneous SNR of the $u$ th branch $\gamma_{u}$ exceeds $x: A_{u}=\left\{\gamma_{u}>x\right\}$. Since the SC output SNR is the maximum of all the branch SNRs, when at least one branch SNR exceeds $x$, so does the SC output. Therefore, we readily bound the ccdf of the multibranch SC output SNR by

$$
\begin{aligned}
\operatorname{Pr}\left(\gamma_{\mathrm{sc}}>x\right) \geq & \sum_{u=1}^{L} \operatorname{Pr}\left(\gamma_{u}>x\right)-\sum_{\substack{u, v=1 \\
u<v}}^{L} \operatorname{Pr}\left(\gamma_{u}>x, \gamma_{v}>x\right) \\
\operatorname{Pr}\left(\gamma_{\mathrm{sc}}>x\right) \leq & \sum_{u=1}^{L} \operatorname{Pr}\left(\gamma_{u}>x\right)-\sum_{\substack{u, v=1 \\
u<v}}^{L} \operatorname{Pr}\left(\gamma_{u}>x, \gamma_{v}>x\right) \\
& +\sum_{\substack{u, v, w=1 \\
u<v<w}}^{L} \operatorname{Pr}\left(\gamma_{u}>x, \gamma_{v}>x, \gamma_{w}>x\right)
\end{aligned}
$$

where $\operatorname{Pr}\left(\gamma_{u}>x\right)$ is the probability that any single branch SNR exceeds $x$, and $\operatorname{Pr}\left(\gamma_{u}>x, \gamma_{v}>x\right)$ is the probability that any two branch SNRs exceed $x$ simultaneously, which are given, respectively, by [2, Eqs. (10-4-8, A-7-1)]

$$
\begin{aligned}
\operatorname{Pr}\left(\gamma_{u}\right. & >x) \\
& =e^{-\frac{x}{\bar{\gamma}_{u}}} \\
\operatorname{Pr}\left(\gamma_{u}\right. & \left.>x, \gamma_{v}>x\right) \\
& =e^{-\frac{x}{\bar{\gamma}_{u}}}\left[1-Q\left(\sqrt{\frac{2 x}{\left(1-\rho_{u v}^{2}\right) \bar{\gamma}_{v}}}, \rho_{u v} \sqrt{\frac{2 x}{\left(1-\rho_{u v}^{2}\right) \bar{\gamma}_{u}}}\right)\right] \\
& +e^{-\frac{x}{\bar{\gamma}_{v}}} Q\left(\rho_{u v} \sqrt{\frac{2 x}{\left(1-\rho_{u v}^{2}\right) \bar{\gamma}_{v}}}, \sqrt{\frac{2 x}{\left(1-\rho_{u v}^{2}\right) \bar{\gamma}_{u}}}\right)
\end{aligned}
$$

where $\rho_{u v}=\psi_{u v} / \sqrt{\psi_{u u} \psi_{v v}}$ is the fading correlation between the $u$ th and the $v$ th branches, and $\operatorname{Pr}\left(\gamma_{u}>x, \gamma_{v}>x, \gamma_{w}>x\right)$ is the probability that any three branch SNRs exceed $x$ simultaneously, which can be derived using (5) as

$$
\begin{aligned}
\operatorname{Pr}\left(\gamma_{u}>x, \gamma_{v}>x, \gamma_{w}>x\right) \\
=\frac{\operatorname{det} \boldsymbol{\Phi}}{\phi_{u u} \phi_{v v} \phi_{w w}} \sum_{k=0}^{\infty} \varepsilon_{k}(-1)^{k} \cos k\left(\chi_{u v}+\chi_{v w}+\chi_{w u}\right) \\
\quad \times \sum_{l, m, n=0}^{\infty} C \nu_{u v}^{l+\frac{k}{2}} \nu_{v w}^{m+\frac{k}{2}} \nu_{w u}^{n+\frac{k}{2}} \Gamma\left(\delta_{1}, d_{u} x\right) \Gamma\left(\delta_{2}, d_{v} x\right) \\
\quad \times \Gamma\left(\delta_{3}, d_{w} x\right)
\end{aligned}
$$

where $d_{u}=\phi_{u u} \psi_{u u} / \overline{\gamma_{u}}$ will be used in the following, and $\Gamma(a, z)$ is the complementary incomplete gamma function. 


\section{Moments of the Three-Branch EGC Output SNR}

A combiner's output moments can be used as alternative performance measures to the conventional error-rate analysis. However, a single moment, such as the mean SNR, is not sufficiently informative, and higher order moments can furnish additional information for system design. For example, the Chebyshev inequality yields $\operatorname{Pr}(|X-\mu|>t)<\sigma_{X}^{2} / t^{2}$, where $E(X)=\mu$. Thus, if $X$ is taken to be the output of a diversity combiner, the variability of the outputs is indicated by the variance. The new expression (13) enables us to evaluate the moments of the output SNR of a three-branch EGC system, the output of which can be written as

$$
\gamma_{\text {egc }}=\frac{\left(r_{1}+r_{2}+r_{3}\right)^{2} E_{s}}{3 N_{0}}
$$

where $E_{s}$ is the transmitted signal energy and $N_{0}$ is the noise power spectral density per branch. The moments of output SNR can be obtained as

$$
\begin{aligned}
E\left(\gamma_{\text {egc }}^{n}\right)= & \left(\frac{E_{s}}{3 N_{0}}\right)^{n} E\left[\left(r_{1}+r_{2}+r_{3}\right)^{2 n}\right] \\
= & \left(\frac{\overline{\gamma_{1}}}{3 \psi_{11}}\right)^{n} \sum_{\begin{array}{c}
k_{1}, k_{2}, k_{3}=0 \\
k_{1}+k_{2}+k_{3}=2 n
\end{array}}^{2 n} \frac{(2 n) !}{k_{1} ! k_{2} ! k_{3} !} \\
& \times E\left(r_{1}^{k_{1}} r_{2}^{k_{2}} r_{3}^{k_{3}}\right)
\end{aligned}
$$

where $E\left(r_{1}^{k_{1}} r_{2}^{k_{2}} r_{3}^{k_{3}}\right)$ can be computed using (13). To the best of the authors' knowledge, (27) is a new result and provides high-order moments of the three-branch EGC output SNR for the most general case.

\section{Output mgf of Three-Branch GSC}

$\operatorname{GSC}(M, L)$ achieves a good tradeoff between performance and implementation complexity [27], [28]. However, very few theoretical results are known for the GSC performance in correlated fading channels. The only published paper dealing with this topic is Mallik and Win [29], who analyze the performance of $\operatorname{GSC}(M, L)$ in equally correlated Nakagami fading. The distribution theory for order statistics of arbitrarily correlated RVs is not fully developed [26]. Our new result (6) enables the performance analysis of three-branch GSC in arbitrarily correlated Rayleigh fading channels.

Since GSC $(1,3)$ and GSC $(3,3)$ are simply SC and MRC, these cases are not treated here. Instead, we consider the $\operatorname{GSC}(2,3)$ system, which combines the largest two branch SNRs to form the output

$$
\gamma_{\mathrm{gsc}}=\gamma_{(2)}+\gamma_{(3)}
$$

where $\gamma_{(1)} \leq \gamma_{(2)} \leq \gamma_{(3)}$. We derive the joint cdf of $\gamma_{(2)}$ and $\gamma_{(3)}$ via the first principles as

$$
\begin{aligned}
F_{\gamma_{(2)}, \gamma_{(3)}}(\alpha, \beta)= & \operatorname{Pr}\left(\gamma_{1} \leq \beta, \gamma_{2} \leq \alpha, \gamma_{3} \leq \alpha\right) \\
& +\operatorname{Pr}\left(\gamma_{1} \leq \alpha, \gamma_{2} \leq \beta, \gamma_{3} \leq \alpha\right) \\
& +\operatorname{Pr}\left(\gamma_{1} \leq \alpha, \gamma_{2} \leq \alpha, \gamma_{3} \leq \beta\right) \\
& -2 \operatorname{Pr}\left(\gamma_{1} \leq \alpha, \gamma_{2} \leq \alpha, \gamma_{3} \leq \alpha\right)
\end{aligned}
$$

where $\beta \geq \alpha>0$.
Applying (6) and differentiating (29) with respect to $\alpha$ and $\beta$ yields the joint pdf of $\gamma_{(2)}$ and $\gamma_{(3)}$ as

$$
\begin{aligned}
p_{\gamma_{(2)}, \gamma_{(3)}}(x, y)= & \frac{\operatorname{det} \boldsymbol{\Phi}}{\phi_{11} \phi_{22} \phi_{33}} \sum_{k=0}^{\infty} \varepsilon_{k}(-1)^{k} \cos (k \chi) \\
& \times \sum_{\substack{l, m, n=0 \\
\infty} \nu_{12}^{l+\frac{k}{2}} \nu_{23}^{m+\frac{k}{2}} \nu_{31}^{n+\frac{k}{2}}}^{3} \\
& \times \sum_{\substack{u, v, w=1 \\
u \neq v \neq w}}^{3} \gamma\left(\delta_{w}, d_{w} x\right) d_{u}^{\delta_{u}} d_{v}^{\delta_{v}} \\
& \times\left[x^{\delta_{u}-1} y^{\delta_{v}-1} e^{-\left(x d_{u}+y d_{v}\right)}\right]
\end{aligned}
$$

where $y \geq x>0$.

Using (30), we can obtain the output mgf of $\operatorname{GSC}(2,3)$ as

$$
\begin{aligned}
M_{\mathrm{gsc}}(s)= & E\left(e^{-s \gamma_{\mathrm{gsc}}}\right) \\
= & \int_{0}^{\infty} \int_{x}^{\infty} p_{\gamma_{(2)}, \gamma_{(3)}}(x, y) e^{-(x+y) s} d y d x \\
= & \frac{\operatorname{det} \boldsymbol{\Phi}}{\phi_{11} \phi_{22} \phi_{33}} \sum_{k=0}^{\infty} \varepsilon_{k}(-1)^{k} \cos (k \chi) \\
& \times \sum_{\substack{l, m, n=0\\
}}^{\infty} C \nu_{12}^{l+\frac{k}{2}} \nu_{23}^{m+\frac{k}{2}} \nu_{31}^{n+\frac{k}{2}} \\
& \times \sum_{\substack{u, v, w=1 \\
u \neq v \neq w}}^{3}\left(\frac{d_{v}}{d_{v}+s}\right)^{\delta_{v}} d_{u}^{\delta_{u}} g(u, v, w)
\end{aligned}
$$

where

$$
\begin{aligned}
g(u, v, w)= & \int_{0}^{\infty} x^{\delta_{u}-1} e^{-x\left(d_{u}+s\right)} \gamma\left(\delta_{w}, x d_{w}\right) \\
& \times \Gamma\left[\delta_{v},\left(d_{v}+s\right) x\right] d x \\
= & \frac{d_{w}^{\delta_{w}}}{\delta_{w}}\left[\frac{\left(\delta_{u}+\delta_{w}-1\right) !\left(\delta_{v}-1\right) !}{\left(d_{u}+d_{w}+s\right)^{\delta_{u}+\delta_{w}}}\right. \\
& \times{ }_{2} F_{1}\left(\delta_{u}+\delta_{w}, 1 ; \delta_{w} ; \frac{d_{w}}{d_{w}+d_{u}+s}\right) \\
& +\frac{(\delta-1) !\left(d_{v}+s\right)^{\delta_{v}}}{\delta_{v}(d+2 s)^{\delta}} \\
& \times F_{A}\left(\delta ; 1,1 ; \delta_{w}+1, \delta_{v}+1 ; \frac{d_{w}}{d+2 s}\right. \\
& \left.\left.\frac{d_{v}+s}{d+2 s}\right)\right]
\end{aligned}
$$

where ${ }_{2} F_{1}(a, b ; c ; z)$ is the Gauss hypergeometric function, which is defined as [30, eq. (9.100)] and $F_{A}\left(\alpha ; \beta_{1}, \ldots, \beta_{n} ; \gamma_{1}, \ldots, \gamma_{n} ; z_{1}, \ldots, z_{n}\right)$ is the $n$ th-order Appell hypergeometric function [30, (9.180.2)]. Equation (32) follows from [30, eq. (9.236.4)] and [31, eq. (C.1)]. Using the output mgf (31), the performance of various digital modulations with GSC $(2,3)$ may be evaluated.

\section{NUMERICAL RESULTS}

Consider a linear array of three antennas in a base station [32]. Assume that the antenna is $h=100 \mathrm{ft}$ high, operating at 


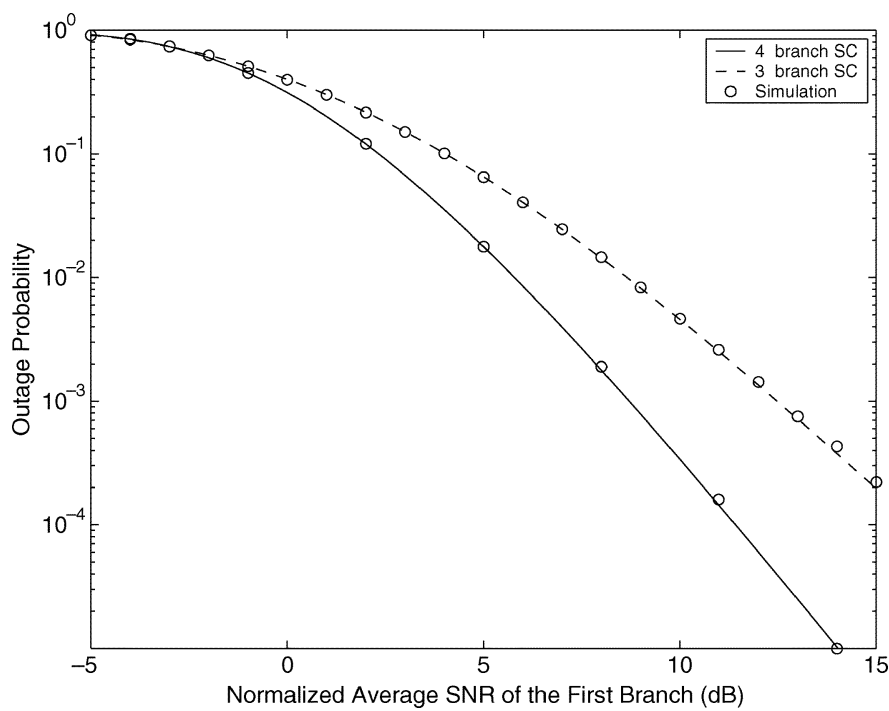

Fig. 1. Outage probability $P_{\text {out }}$ of SC versus the normalized average branch SNR $\bar{\gamma} / \gamma_{\text {th }}$ in correlated Rayleigh fading channel.

$850 \mathrm{MHz}$ (i.e., wavelength is $1.16 \mathrm{ft}$ ). The antenna spacing is $d=10 \times 1.16=11.6 \mathrm{ft}$, and the mean angle of arrival (AOA) is $\phi_{0}=30^{\circ}$. The normalized envelope covariance matrix of the linear array is determined using empirical curves [33] as

$$
\Psi=\left(\begin{array}{ccc}
1 & 0.61 & 0.37 \\
0.61 & 1 & 0.61 \\
0.37 & 0.61 & 1
\end{array}\right)
$$

Using [6, eq. (1.5-26)], (33) can be converted to the covariance matrix of the underlying Gaussian RVs $\Psi$. The outage probability of three-branch SC in Rayleigh fading channel with this linear antenna array is plotted in Fig. 1.

Consider an antenna array with normalized covariance matrix

$$
\Psi=\left(\begin{array}{llll}
1.0000 & 0.2920 & 0.2998 & 0.1121 \\
0.2920 & 0.6602 & 0.2031 & 0.1585 \\
0.2998 & 0.2031 & 0.7625 & 0.1888 \\
0.1121 & 0.1585 & 0.1888 & 0.6431
\end{array}\right)
$$

The inverse covariance matrix now satisfies (15). Thus, using (19), we may evaluate the outage probability of four-branch SC, as is shown in Fig. 1. Semianalytical simulation results are provided as an independent check of our numerical results. We use the Cholesky decomposition approach [34] to generate correlated complex Gaussian variables, and their amplitudes give the required correlated Rayleigh envelopes. Our numerical results agree with the semianalytical simulation results.

Fig. 2 shows the impact of an exponentially decaying power delay profile $\left(\overline{\gamma_{l}}=e^{-\eta(l-1)} \overline{\gamma_{1}}, l=1,2,3\right.$, where $\eta$ is the

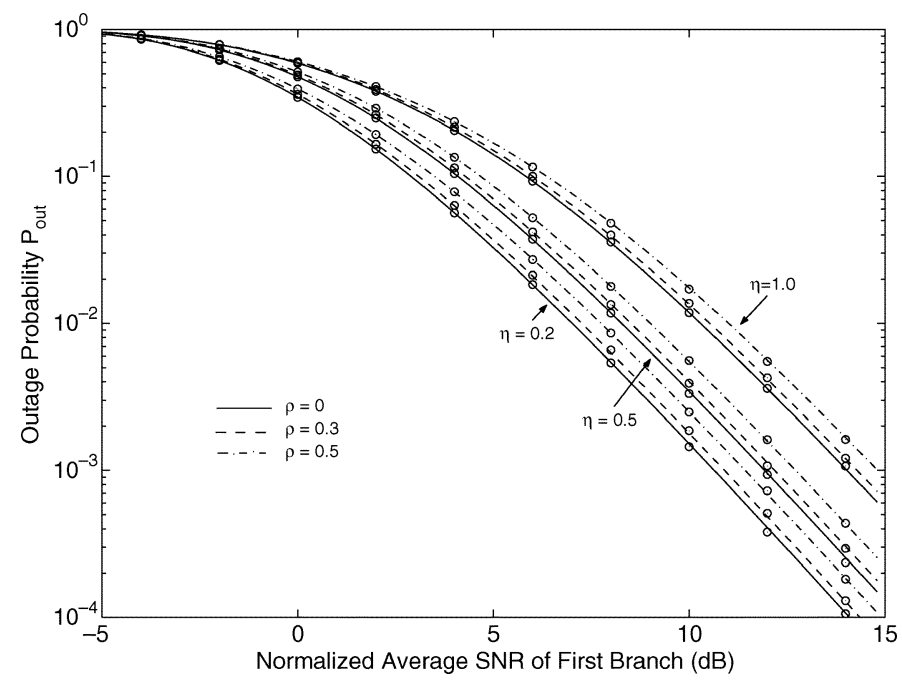

Fig. 2. Outage probability $P_{\text {out }}$ of three-branch SC versus normalized average SNR of the first branch $\bar{\gamma}_{1} / \gamma_{\text {th }}$ over exponentially correlated Rayleigh fading channel.

power decay factor) [35] and an exponentially correlated Rayleigh fading on the outage probability of three-branch SC. As expected, the performance of three-branch SC degrades as the power decay factor $\eta$ or the branch correlation $\rho$ increases. That is, both the branch SNR imbalance and fading correlation degrade the performance of diversity systems. This observation agrees with that in [35].

Clarke [36] derived the relationship between envelope correlation and antenna separation assuming a uniform AOA distribution. However, the measurement data in [37] shows that a Gaussian AOA distribution is more realistic than the uniform AOA distribution for GSM systems in rural and suburban areas. Thus, we consider a linear antenna array of five vertical omnidirectional antennas, with antenna spacing of $80 \%$ of the wavelength. The AOA is assumed to be Gaussian distributed [38] with mean $\phi_{0}=30^{\circ}$ and variance (angular spread) $\sigma_{\phi}=$ $10^{\circ}$. The covariance matrix of the underlying complex Gaussian components can be computed using [39, eq. $(33,34)]$ (see $(35)$, shown at the bottom of the page).

Fig. 3 shows that our bounds fit the simulation results well in the high-SNR region. In this particular case, the lower bound is more accurate than the upper bound. The tightness of our bounds is subject to the covariance matrix. We also find that these bounds for an equally correlated system are tight. From (21) and (22), the exact outage probability can be dominated by first- and second-order events. Particularly, for high SNR, this appears to be the case. That the lower bound includes all the first- and second-order events contributes its tightness. However, the upper bound in Fig. 3 breaks down for SNRs less than

$$
\Psi=\bar{\gamma}\left(\begin{array}{ccccc}
1 & -0.6+0.45 i & 0.13-0.3 i & -0.02+0.08 i & 0.01-0.01 i \\
-0.6-0.45 i & 1 & -0.6+0.45 i & 0.13-0.3 i & -0.02+0.08 i \\
0.13+0.3 i & -0.6-0.45 i & 1 & -0.6+0.45 i & 0.13-0.3 i \\
-0.02-0.08 i & 0.13+0.3 i & -0.6-0.45 i & 1 & -0.6+0.45 i \\
0.01+0.01 i & -0.02-0.08 i & 0.13+0.3 i & -0.6-0.45 i & 1
\end{array}\right)
$$




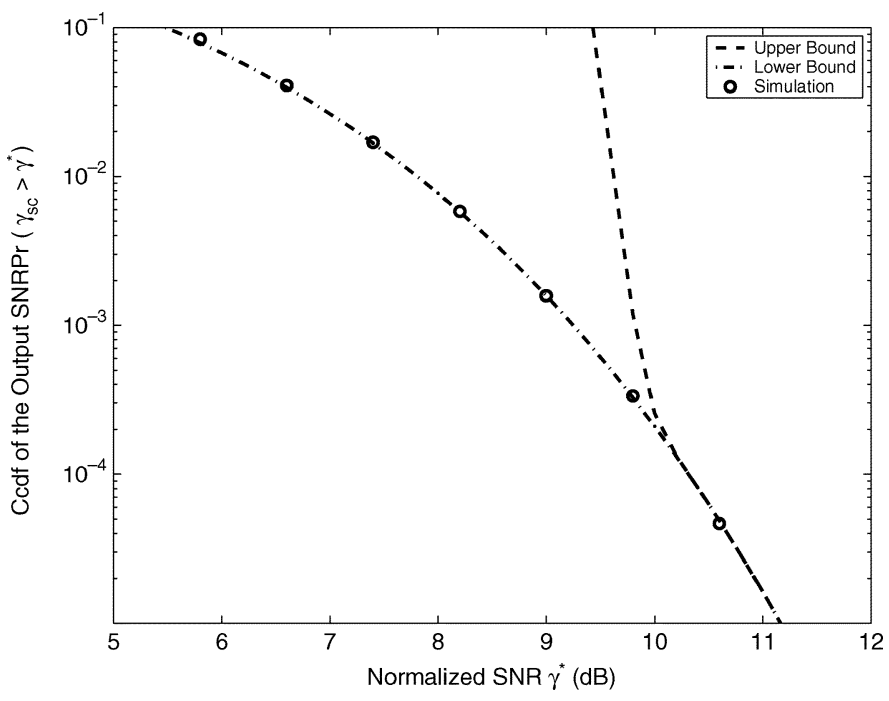

Fig. 3. Bounds of output ccdf of five-branch SC.

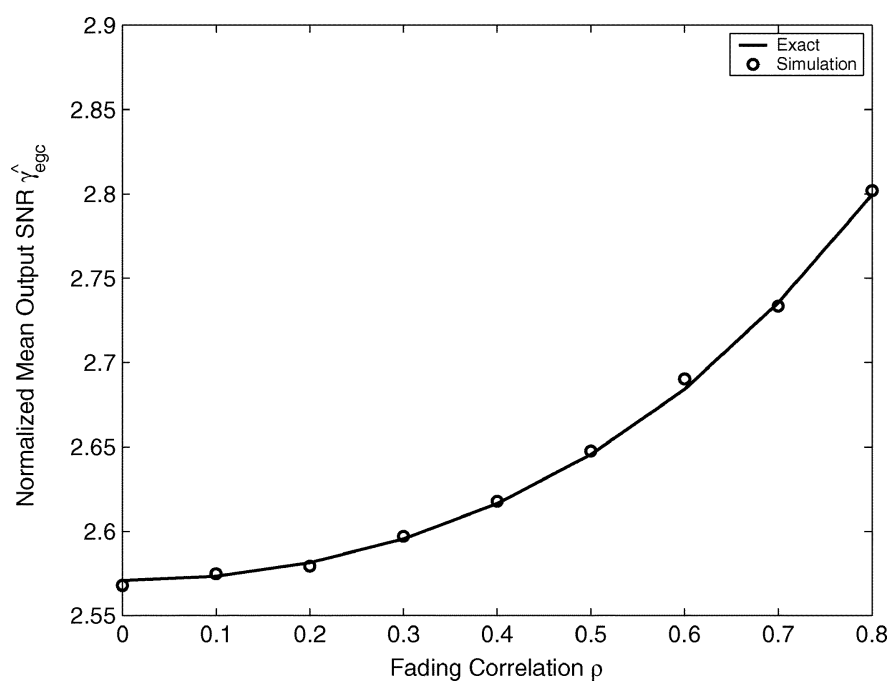

Fig. 4. Mean output SNR of three-branch EGC normalized by the first branch SNR.

$10 \mathrm{~dB}$. The reason is that there are a large number of third-order events [see (23)], and their probability sum can be quite large.

Fig. 4 shows the effect of fading correlation on the normalized mean output SNR of three-branch EGC. As fading correlation increases, the mean output SNR also increases. This contradicts the conventional wisdom that diversity combiner performance degrades with increasing correlation. However, it can be explained by (27). Since $E\left(R_{j} R_{k}\right)>E\left(R_{j}\right) E\left(R_{k}\right),(j \neq$ $k$ ), for $\rho>0$, the average output SNR of EGC in correlated fading channels is higher than that in independent fading channels [40]. As $\rho$ approaches one, the average output SNR of EGC approaches that of MRC. It should be noted that common EGC performance measures, such as bit-error rate (BER), cannot be solely characterized by the average output SNR; they also depend on the higher moments. The average output SNR by itself is not a comprehensive metric for the EGC performance in correlated fading channels. Caution must therefore be exercised when using the average output SNR as a performance measure, and one should consider the higher order moments of the combiner output SNR [41].

\section{CONCLUSION}

In this paper, using Miller's classical approach, we have derived the new infinite series representations for the joint pdf and the joint cdf of three and four correlated Rayleigh RVs. Several previous results have turned out to be special cases of our new results. Our results are canonical, in that they can accommodate any arbitrary $3 \times 3$ correlation matrix, as well as a fairly general class of $4 \times 4$ correlation matrices. These representations pave the way for solving certain long-standing diversity problems. For example, the performance of three-branch SC, EGC, and GSC in arbitrarily correlated Rayleigh fading can now be evaluated analytically. To the best of our knowledge, these have never been developed up to now. Similarly, the performance of four-branch diversity combiners in a fairly general correlation setting can now be completely solved using our results. Our novel use of Bonferroni's inequality allows the bounding of the multibranch SC performance for arbitrary correlation structures among the diversity branches. Other applications may include finding transition probabilities for Markov modeling of the Rayleigh fading channel [42]. Finally, theoretical performance results of diversity systems in correlated fading are very scarce, compared with that of independent fading. The results derived in this paper partly redress this issue, while much more work remains to be done.

\section{APPENDIX A \\ DERIVATION OF (16)}

Let $\boldsymbol{G}=\left\{G_{1}, G_{2}, G_{3}, G_{4}\right\}$ be jointly complex Gaussian RVs with zero means and the inverse covariance matrix $\Phi$ given by (15). The joint pdf $p_{\boldsymbol{R}, \Theta}(\boldsymbol{r}, \boldsymbol{\theta})$ of the corresponding amplitudes $\boldsymbol{R}$ and phases $\boldsymbol{\Theta}$ of $\boldsymbol{G}$ is given by [3]

$$
p_{\boldsymbol{R}, \Theta}(\boldsymbol{r}, \boldsymbol{\theta})=\frac{1}{\pi^{4}}|\operatorname{det}(\boldsymbol{\Phi})| r_{1} r_{2} r_{3} r_{4} e^{-\boldsymbol{g}^{*} \boldsymbol{\Phi} \boldsymbol{g}}
$$

where $\boldsymbol{g}=\boldsymbol{r} e^{j \boldsymbol{\theta}}$. The marginal density $p_{\boldsymbol{R}}(\boldsymbol{r})$ can therefore be obtained

$$
\begin{aligned}
p_{\boldsymbol{R}}\left(r_{1}, r_{2}, r_{3}, r_{4}\right)= & \frac{1}{\pi^{4}}|\operatorname{det} \boldsymbol{\Phi}| r_{1} r_{2} r_{3} r_{4} \\
& \times \int_{0}^{2 \pi} \int_{0}^{2 \pi} \int_{0}^{2 \pi} \int_{0}^{2 \pi} e^{-\boldsymbol{g}^{*} \boldsymbol{\Phi} \boldsymbol{g}} d \theta_{1} d \theta_{2} d \theta_{3} d \theta_{4} .
\end{aligned}
$$

Writing $G_{k}$ in terms of polar coordinates, we obtain

$$
\begin{aligned}
\boldsymbol{g}^{*} \boldsymbol{\Phi} \boldsymbol{g}= & r_{1}^{2} \phi_{11}+r_{2}^{2} \phi_{22}+r_{3}^{2} \phi_{33}+r_{4}^{2} \phi_{44} \\
& +2 r_{1} r_{2}\left|\phi_{12}\right| \cos \left(\theta_{1}-\theta_{2}-\chi_{12}\right) \\
& +2 r_{1} r_{3}\left|\phi_{13}\right| \cos \left(\theta_{1}-\theta_{3}-\chi_{13}\right) \\
& +2 r_{2} r_{3}\left|\phi_{23}\right| \cos \left(\theta_{2}-\theta_{3}-\chi_{23}\right) \\
& +2 r_{2} r_{4}\left|\phi_{24}\right| \cos \left(\theta_{2}-\theta_{4}-\chi_{24}\right) \\
& +2 r_{3} r_{4}\left|\phi_{34}\right| \cos \left(\theta_{3}-\theta_{4}-\chi_{34}\right) .
\end{aligned}
$$


Making variable transforms $\theta_{1}-\theta_{2}=u, \theta_{2}-\theta_{3}=v$, and $\theta_{3}-\theta_{4}=w$, and substituting (38) into (37), we obtain the pdf of $R$ as

$$
\begin{aligned}
& p_{\boldsymbol{R}}\left(r_{1}, r_{2}, r_{3}, r_{4}\right) \\
& =\frac{2}{\pi^{3}}|\operatorname{det} \Phi| r_{1} r_{2} r_{3} r_{4} e^{-\left(r_{1}^{2} \phi_{11}+r_{2}^{2} \phi_{22}+r_{3}^{2} \phi_{33}+r_{4}^{2} \phi_{44}\right)} \\
& \quad \times \int_{0}^{2 \pi} \int_{0}^{2 \pi} \int_{0}^{2 \pi} e^{-2 r_{2} r_{3}\left|\phi_{23}\right| \cos \left(v-\chi_{23}\right)} e^{-\left(a_{3} \cos w+a_{4} \sin w\right)} \\
& \quad \times e^{-\left(a_{1} \cos u+a_{2} \sin u\right)} d u d v d w
\end{aligned}
$$

where $a_{1}, a_{2}, a_{3}$, and $a_{4}$ depend on $v$, but not on $u$ and $w$

$$
\begin{aligned}
& a_{1}=2 r_{1} r_{2}\left|\phi_{12}\right| \cos \chi_{12}+2 r_{1} r_{3}\left|\phi_{13}\right| \cos \left(v-\chi_{13}\right) \\
& a_{2}=2 r_{1} r_{2}\left|\phi_{12}\right| \sin \chi_{12}-2 r_{1} r_{3}\left|\phi_{13}\right| \sin \left(v-\chi_{13}\right) \\
& a_{3}=2 r_{3} r_{4}\left|\phi_{34}\right| \cos \chi_{34}+2 r_{2} r_{4}\left|\phi_{24}\right| \cos \left(v-\chi_{24}\right) \\
& a_{4}=2 r_{3} r_{4}\left|\phi_{34}\right| \sin \chi_{34}-2 r_{2} r_{4}\left|\phi_{24}\right| \cos \left(v-\chi_{24}\right) .
\end{aligned}
$$

Using the relationship [3]

$$
\int_{0}^{2 \pi} \exp [-(a \cos \theta+b \sin \theta)] d \theta=2 \pi I_{0}\left(\sqrt{a^{2}+b^{2}}\right)
$$

and Neumann's addition theorem [43, p. 365], we obtain the joint pdf of quadrivariate Rayleigh distribution as (42)

$$
\begin{aligned}
& p_{\boldsymbol{R}}\left(r_{1}, r_{2}, r_{3}, r_{4}\right) \\
& =\frac{4}{\pi}(\operatorname{det} \boldsymbol{\Phi}) r_{1} r_{2} r_{3} r_{4} \\
& \quad \times \exp \left[-\left(r_{1}^{2} \phi_{11}+r_{2}^{2} \phi_{22}+r_{3}^{2} \phi_{33}+r_{4}^{2} \phi_{44}\right)\right] \\
& \quad \times \sum_{m=0}^{\infty} \sum_{n=0}^{\infty} \varepsilon_{m} \varepsilon_{n} I_{m}\left(2 r_{1} r_{2}\left|\phi_{12}\right|\right) I_{m}\left(2 r_{1} r_{3}\left|\phi_{13}\right|\right) \\
& \quad \times I_{n}\left(2 r_{2} r_{4}\left|\phi_{24}\right|\right) I_{n}\left(2 r_{3} r_{4}\left|\phi_{34}\right|\right) \\
& \quad \times \int_{0}^{2 \pi} e^{-2 r_{2} r_{3}\left|\phi_{23}\right| \cos \left(v-\chi_{23}\right)} \\
& \quad \times\left\{\cos \left[m\left(v+\chi_{12}-\chi_{13}\right)+n\left(v+\chi_{34}-\chi_{24}\right)\right]\right. \\
& \quad+\cos \left[m\left(v+\chi_{12}-\chi_{13}\right)\right. \\
& \left.\left.\quad-n\left(v+\chi_{34}-\chi_{24}\right)\right]\right\} d v .
\end{aligned}
$$

Using the relation [19, eq. (3-3.14)], we obtain (16) after algebraic manipulation.

\section{REFERENCES}

[1] W. R. Young, "Comparison of mobile radio transmission at 150, 450, 900, and 3700 MHz," Bell Syst. Tech. J., vol. 31, pp. 1068-1085, 1952.

[2] M. Schwartz, W. R. Bennett, and S. Stein, Communication Systems and Techniques. New York: McGraw-Hill, 1966.

[3] K. S. Miller, "Complex Gaussian processes," SIAM, vol. 11, pp. 544-567, Oct. 1969

[4] L. E. Blumenson and K. S. Miller, "Properties of generalized Rayleigh distributions," Ann. Math. Statist., vol. 34, pp. 903-910, 1963.

[5] A. A. Abu-Dayya and N. C. Beaulieu, "Analysis of switched diversity systems on generalized fading channels," IEEE Trans. Commun., vol. 42, no. 11, pp. 2959-2966, Nov. 1994.
[6] W. C. Jakes, Microwave Mobile Communications. New York: IEEE Press, 1994.

[7] M. K. Simon and M.-S. Alouini, Digital Communication Over Fading Channels, 1st ed. New York: Wiley, 2000.

[8] C. Tellambura and V. K. Bhargava, "A performance analysis of trellis coded modulation schemes in nonindependent Rician fading channels," in Proc. Global Telecommun. Conf., vol. 3, 1993, pp. 1424-1428.

[9] S. O. Rice, "Mathematical analysis of random noise," Bell Syst. Tech. J., vol. 23, pp. 282-332, 1944.

[10] C. C. Tan and N. C. Beaulieu, "Infinite series representations of the bivariate Rayleigh and Nakagami- $m$ distributions," IEEE Trans. Commun., vol. 45, no. 10, pp. 1159-1161, Oct. 1997.

[11] C. Tellambura, A. Annamalai, and V. K. Bhargava, "Closed-form and infinite series solutions for the MGF of a dual-diversity selection combiner output in bivariate Nakagami fading," IEEE Trans. Commun., vol. 51, no. 4, pp. 539-542, Apr. 2003.

[12] R. Mallik, M. Win, and J. Winters, "Performance of dual-diversity predetection EGC in correlated Rayleigh fading with unequal branch SNRs," IEEE Trans. Commun., vol. 50, no. 7, pp. 1041-1044, Jul. 2002.

[13] A. Annamalai, V. Ramanathan, and C. Tellambura, "Analysis of equal-gain diversity receiver in correlated fading channels," in Proc. Veh. Technol. Conf., vol. 4, May 2002, pp. 2038-2041.

[14] G. K. Karagiannidis, D. A. Zogas, and S. A. Kotsopoulos, "On the multivariate Nakagami- $m$ distribution with exponential correlation," IEEE Trans. Commun., vol. 51, no. 8, pp. 1240-1244, Aug. 2003.

[15] — "Performance analysis of triple selection diversity over exponentially correlated Nakagami- $m$ fading channels," IEEE Trans. Commun., vol. 51, no. 8, pp. 1245-1248, Aug. 2003.

[16] G. K. Karagiannidis, D. A. Zogas, and S. A. Kotsopoulos, "An efficient approach to multivariate Nakagami- $m$ distribution using Green's matrix approximation," IEEE Trans. Wireless Commun., vol. 2, no. 5, pp. 883-889, Sep. 2003.

[17] D. A. Zogas, G. K. Karagiannidis, and S. A. Kotsopoulos, "On the average output SNR in selection combining with three correlated brances over Nakagami-m fading channels," IEEE Trans. Wireless Commun., vol. 3, no. 1, pp. 25-28, Jan. 2004.

[18] Y. Chen and C. Tellambura, "Performance analysis of three-branch selection combining over arbitrarily correlated Rayleigh fading channels," IEEE Trans. Wireless Commun., vol. 4, no. 3, pp. 861-865, May 2005.

[19] K. S. Miller, Complex Scochastic Processes. Reading, MA: AddisonWesley, 1974.

[20] M. Abramowitz and I. A. Stegun, Handbook of Mathematical Functions With Formulas, Graphs, and Mathematical Tables. New York: Dover, 1972.

[21] V. A. Aalo, "Performance of maximal-ratio diversity systems in a correlated Nakagami-fading environment," IEEE Trans. Commun., vol. 43, no. 8 , pp. 2360-2369, Aug. 1995.

[22] J. G. Proakis, Digital Communications, 3rd ed. New York: McGrawHill, 1995.

[23] A. Annamalai, C. Tellambura, and V. K. Bhargava, "Simple and accurate methods for outage analysis in cellular mobile radio systems-A unified approach," IEEE Trans. Commun., vol. 49, no. 2, pp. 303-316, Feb. 2001

[24] O. C. Ugweje and V. A. Aalo, "Performance of selection diversity system in correlated Nakagami fading," in Proc. IEEE Veh. Technol. Conf., vol. 3, New York, NY, 1997, pp. 1488-1492.

[25] Q. T. Zhang and H. G. Lu, "A general analytical approach to multibranch selection combining over various spatially correlated fading channels," IEEE Trans. Commun., vol. 50, no. 7, pp. 1066-1073, Jul. 2002.

[26] H. A. David, Order Statistics, 2nd ed. New York: Wiley, 1981.

[27] N. Kong, T. Eng, and L. B. Milstein, "A selection combining scheme for RAKE receivers," in Proc. IEEE Int. Conf. Universal Pers. Commun., 1995, pp. 426-430.

[28] L. Yue, "Analysis of generalized selection combining techniques," in Proc. IEEE Veh. Technol. Conf., vol. 2, 2000, pp. 1191-1195.

[29] R. K. Mallik and M.Z. Win, "Analysis of hybrid selection/maximal-ratio combining in correlated Nakagami fading," IEEE Trans. Commun., vol. 50, no. 8, pp. 1372-1383, Aug. 2002.

[30] I. S. Gradshteyn and I. M. Ryzhik, Table of Integrals, Series, and Products, 5th ed. New York: Academic, 1994.

[31] A. Annamalai, C. Tellambura, and V. K. Bhargava, "Equal-gain diversity receiver performance in wireless channels," IEEE Trans. Commun., vol. 48, no. 10 , pp. $1732-1745$, Oct. 2000.

[32] Q. T. Zhang, "Maximal-ratio combining over Nakagami fading channels with an arbitrary branch covariance matrix," IEEE Trans. Veh. Technol., vol. 48, no. 4, pp. 1141-1150, Jul. 1999. 
[33] W. C. Y. Lee, Mobile Communications: Design Fundamentals. New York: Wiley, 1993.

[34] M. C. Jeruchim, P. Balaban, and K. S. Shanmugan, Simulation of Communication Systems: Modeling, Methodology and Techniques, 2nd ed. New York: Kluwer/Plenum, 2000.

[35] Y.-C. Ko, M.-S. Alouini, and M. K. Simon, "Outage probability of diversity systems over generalized fading channels," IEEE Trans. Commun., vol. 48, no. 11, pp. 1783-1787, Nov. 2000.

[36] R. H. Clarke, "A statistical theory of mobile radio reception," Bell Syst. Tech. J., vol. 47, pp. 957-1000, 1968.

[37] D. Aszetly, "On antenna arrays in mobile communication systems: Fast fading and GSM base station receiver algorithm," Ph.D. dissertation, Royal Inst. Technol., Stockholm, Sweden, Mar. 1996.

[38] J. Salz and J. H. Winters, "Effect of fading correlation on adaptive arrays in digital mobile radio," IEEE Trans. Veh. Technol., vol. 43, no. 6, pp. 1049-1057, Nov. 1994.

[39] J. Luo, J. R. Zeidler, and S. McLaughlin, "Performance analysis of compact antenna arrays with MRC in correlated Nakagami fading channels," IEEE Trans. Veh. Technol., vol. 50, no. 1, pp. 267-277, Jan. 2001.

[40] D. G. Brennan, "Linear diversity combining techniques," Proc. IRE, vol. 47, pp. 1075-1102, Jun. 1959.

[41] G. K. Karagiannidis, "Moments-based approach to the performance analysis of equal gain diversity in Nakagami- $m$ fading," IEEE Trans. Commun., vol. 52, no. 5, pp. 685-690, May 2004.

[42] J. G. Kemeny and J. L. Snell, Finite Markov Chains. Princeton, NJ: Van Nostrand, 1960.

[43] G. N. Watson, A Treatise on the Theory of Bessel Functions. Cambridge, U.K.: Cambridge Univ. Press, 1944.

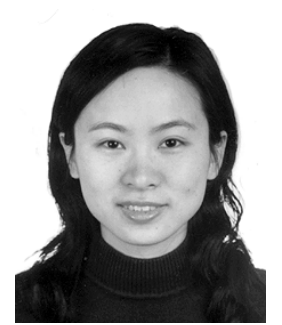

Yunxia Chen (S'03) received the B.Sc. degree in information engineering from Shanghai Jiaotong University, Shanghai, China, in 1998, and the M.Sc. degree from the University of Alberta, Edmonton, $\mathrm{AB}$, Canada, in 2004. She is currently working toward the Ph.D. degree in the Department of Electrical and Computer Engineering, University of California, Davis.

Her research interests are in energy-constrained signal processing and networking techniques, protocol design and performance analysis of wireless sensor networks, diversity techniques and fading channels, and MIMO systems.

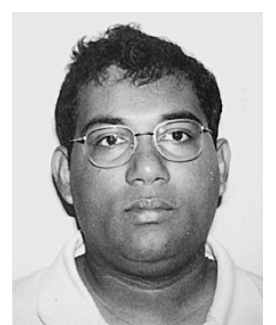

Chinthananda Tellambura (M'97-SM'02) received the B.Sc. degree (with first-class honors) from the University of Moratuwa, Moratuwa, Sri Lanka, in 1986, the M.Sc. degree in electronics from the University of London, London, U.K., in 1988, and the $\mathrm{Ph} . \mathrm{D}$. degree in electrical engineering from the University of Victoria, Victoria, BC, Canada, in 1993.

He was a Postdoctoral Research Fellow with the University of Victoria (1993-1994) and the University of Bradford (1995-1996). He was with Monash University, Melbourne, Australia, from 1997 to 2002. Presently, he is an Associate Professor with the Department of Electrical and Computer Engineering, University of Alberta. His research interests include coding, communication theory, modulation, equalization, and wireless communications.

Prof. Tellambura is an Associate Editor for both the IEEE TRANSACTIONS on Communications and the IEEE TRAnsactions ON Wireless Communications. He is a Co-Chair of the Communication Theory Symposium of Globecom' 05 , to be held in St. Louis, MO. 